\title{
Orbital Alignment for Accurate Projection-Based Embedding Calculations along Reaction Paths
}

\author{
Moritz Bensberg, Johannes Neugebauer ${ }^{1}$ \\ Theoretische Organische Chemie, Organisch-Chemisches Institut \\ and Center for Multiscale Theory and Computation, \\ Westfälische Wilhelms-Universität Münster \\ Corrensstraße 40, 48149 Münster, Germany \\ Supporting Information
}

Date: $\quad$ May 5, 2020

${ }^{1}$ email: j.neugebauer@uni-muenster.de 
Table SI: Reaction energy error for DOS-BLYP-in-BLYP embedding with basis set truncation with respect to the full BLYP calculations.

DOS Threshold $\tau$

\begin{tabular}{lrrrrrrrr}
\hline Reaction & $3.0 \cdot 10^{-2}$ & $2.5 \cdot 10^{-2}$ & $2.0 \cdot 10^{-2}$ & $1.5 \cdot 10^{-2}$ & $1.0 \cdot 10^{-2}$ & $8.0 \cdot 10^{-3}$ & $5.0 \cdot 10^{-3}$ & $1.0 \cdot 10^{-3}$ \\
\hline $\mathbf{( 1 )}$ & 0.046 & 0.028 & 0.028 & -0.116 & -0.114 & -0.114 & -0.023 & -0.002 \\
$(\mathbf{2})$ & -0.419 & -0.349 & -0.355 & -0.119 & 0.020 & 0.020 & -0.000 & -0.002 \\
$(\mathbf{3})$ & -0.310 & -0.300 & -0.300 & 0.050 & -0.048 & -0.048 & -0.058 & 0.002 \\
$(\mathbf{4})$ & 0.616 & -0.438 & -0.289 & 0.039 & 0.039 & 0.032 & 0.007 & 0.001 \\
$(\mathbf{5})$ & -0.092 & -0.090 & 0.076 & -0.012 & 0.042 & 0.035 & -1.949 & -0.005 \\
$(\mathbf{6})$ & 4.451 & 4.451 & 0.205 & 0.526 & -0.027 & -0.104 & -0.067 & -0.007 \\
\hline
\end{tabular}

\section{Basis-Set Truncation Errors}

In order to evaluate the error introduced by basis-set truncation in the reaction barriers and reaction energies, we calculated the relative energy error based on the GGA-in-GGA calculations with respect to the full supersystem calculations. The error is defined as in the main text.

The $\Delta \Delta E$ values for DOS-BLYP-in-BLYP are shown in Tabs. SI and SIII for the reaction energy and reaction barrier, respectively. The errors for DOS-PBE-in-PBE are shown in Tabs. SII and SIV, the errors for MS-BLYP-in-BLYP in Tabs. SV and SVII and for MS-PBE-in-PBE in Tabs. SVI and SVIII.

For the DOS-based calculations, the error is given as a function of the truncation threshold $\tau_{\text {loc }}=\tau_{\text {kin }}=\tau$. For the MS based calculation it is given as a function of the system selection, starting with the smallest active system denoted by 1 and then going to larger active systems as illustrated in Fig. S1. Errors larger than $2 \mathrm{~kJ} \mathrm{~mol}^{-1}$ are highlighted. 
Table SII: Reaction energy error for DOS-PBE-in-PBE embedding with basis set truncation with respect to the full PBE calculations.

DOS Threshold $\tau$

\begin{tabular}{lrrrrrrrr}
\hline Reaction & $3.0 \cdot 10^{-2}$ & $2.5 \cdot 10^{-2}$ & $2.0 \cdot 10^{-2}$ & $1.5 \cdot 10^{-2}$ & $1.0 \cdot 10^{-2}$ & $8.0 \cdot 10^{-3}$ & $5.0 \cdot 10^{-3}$ & $1.0 \cdot 10^{-3}$ \\
\hline$(\mathbf{1})$ & -0.073 & -0.014 & -0.016 & -0.250 & -0.248 & -0.086 & -0.013 & -0.001 \\
$(\mathbf{2})$ & 0.013 & 0.100 & 0.103 & -0.158 & -0.008 & -0.008 & 0.003 & 0.000 \\
$(\mathbf{3})$ & -0.335 & -0.328 & -0.328 & -0.049 & 0.156 & 0.157 & -0.055 & -0.002 \\
$(\mathbf{4})$ & 0.702 & 1.078 & -0.388 & 0.031 & 0.033 & 0.011 & 0.006 & -0.002 \\
$(\mathbf{5})$ & -0.055 & 0.208 & -0.104 & -0.320 & -0.081 & 0.014 & -2.867 & -0.011 \\
$(\mathbf{6})$ & 6.810 & 6.810 & 2.599 & 0.805 & 1.076 & 1.000 & 0.968 & 1.054 \\
\hline
\end{tabular}

Table SIII: Reaction barrier error for DOS-BLYP-in-BLYP embedding with basis set truncation with respect to the full BLYP calculations.

DOS Threshold $\tau$

\begin{tabular}{lrrrrrrrr}
\hline Reaction & $3.0 \cdot 10^{-2}$ & $2.5 \cdot 10^{-2}$ & $2.0 \cdot 10^{-2}$ & $1.5 \cdot 10^{-2}$ & $1.0 \cdot 10^{-2}$ & $8.0 \cdot 10^{-3}$ & $5.0 \cdot 10^{-3}$ & $1.0 \cdot 10^{-3}$ \\
\hline$(\mathbf{1})$ & -0.049 & -0.073 & -0.073 & -0.159 & -0.158 & -0.158 & 0.020 & 0.003 \\
$(\mathbf{2})$ & -0.054 & -0.023 & -0.048 & -0.000 & 0.002 & 0.002 & -0.002 & -0.002 \\
$(\mathbf{3})$ & -0.505 & -0.501 & -0.501 & 0.041 & -0.063 & -0.063 & -0.064 & 0.005 \\
$(\mathbf{4})$ & 0.993 & 0.140 & -0.012 & 0.006 & 0.012 & 0.011 & 0.001 & 0.001 \\
\hline
\end{tabular}


Table SIV: Reaction barrier error for DOS-PBE-in-PBE embedding with basis set truncation with respect to the full PBE calculations.

DOS Threshold $\tau$

\begin{tabular}{lrrrrrrrr}
\hline Reaction & $3.0 \cdot 10^{-2}$ & $2.5 \cdot 10^{-2}$ & $2.0 \cdot 10^{-2}$ & $1.5 \cdot 10^{-2}$ & $1.0 \cdot 10^{-2}$ & $8.0 \cdot 10^{-3}$ & $5.0 \cdot 10^{-3}$ & $1.0 \cdot 10^{-3}$ \\
\hline$(\mathbf{1})$ & -0.180 & -0.130 & -0.090 & -0.158 & -0.158 & -0.007 & 0.024 & 0.002 \\
$(\mathbf{2})$ & 0.405 & 0.451 & 0.436 & -0.032 & -0.029 & -0.029 & -0.002 & -0.002 \\
$(\mathbf{3})$ & -0.576 & -0.572 & -0.572 & -0.114 & -0.006 & -0.006 & -0.066 & -0.003 \\
$(\mathbf{4})$ & 1.107 & 1.040 & -0.170 & -0.002 & 0.004 & -0.002 & -0.003 & -0.006 \\
\hline
\end{tabular}

Table SV: Reaction energy error for MS-BLYP-in-BLYP embedding with basis set truncation with respect to the full BLYP calculations.

\begin{tabular}{lrrrrrrrrr}
\hline \multicolumn{7}{c}{ Selection } \\
\hline Reaction & 1 & 2 & 3 & 4 & 5 & 6 & 7 & 8 \\
\hline$(\mathbf{1})$ & 1.144 & 0.100 & 2.340 & -0.011 & 2.374 & -0.003 & 0.000 & 0.001 \\
$(\mathbf{2})$ & -0.215 & -0.609 & -0.482 & -0.247 & -0.206 & -0.036 & -0.040 & -0.006 \\
$(3)$ & -1.441 & 1.127 & -0.333 & -0.050 & -0.044 & -0.059 & -0.028 & -0.020 \\
$(4)$ & -1.376 & -0.897 & 0.022 & 0.015 & - & - & - & - \\
$(5)$ & 0.300 & 0.670 & 0.299 & 0.370 & -0.018 & - & - & - \\
$(6)$ & -0.252 & 0.055 & 0.023 & - & - & - & - & - \\
\hline
\end{tabular}


Table SVI: Reaction energy error for MS-PBE-in-PBE embedding with basis set truncation with respect to the full PBE calculations.

\begin{tabular}{lrrrrrrrr}
\hline \multicolumn{1}{c}{ Selection } \\
\hline Reaction & 1 & 2 & 3 & 4 & 5 & 6 & 7 & 8 \\
\hline$(\mathbf{1})$ & -0.014 & -0.020 & -0.007 & -0.007 & -0.007 & -0.002 & 0.001 & 0.002 \\
$(\mathbf{2})$ & -0.244 & -0.680 & -0.520 & -0.260 & -0.225 & -0.034 & -0.042 & -0.004 \\
$(3)$ & -1.595 & -0.268 & -0.062 & -0.095 & -0.096 & -0.108 & -0.076 & -0.069 \\
$(4)$ & -1.223 & -0.897 & -0.047 & 0.011 & - & - & - & - \\
$(5)$ & 0.265 & 0.106 & -0.030 & -0.001 & -0.030 & - & - & - \\
$(6)$ & 0.755 & 1.119 & 1.091 & - & - & - & - & - \\
\hline
\end{tabular}

Table SVII: Reaction barrier error for MS-BLYP-in-BLYP embedding with basis set truncation with respect to the full BLYP calculations.

\begin{tabular}{lrrrrrrrrr}
\hline \multicolumn{8}{c}{ Selection } \\
\hline Reaction & 1 & 2 & 3 & 4 & 5 & 6 & 7 & 8 \\
\hline$(\mathbf{1})$ & 1.006 & 1.306 & -0.022 & 1.165 & -0.010 & 1.191 & -0.000 & -0.000 \\
$(\mathbf{2})$ & 0.276 & -0.017 & -0.083 & -0.043 & -0.076 & 0.013 & -0.021 & -0.003 \\
$(3)$ & -1.579 & 0.785 & -0.594 & 0.044 & 0.020 & 0.015 & 0.004 & 0.006 \\
$(4)$ & -1.515 & -0.545 & 0.378 & 0.009 & - & - & - & - \\
\hline
\end{tabular}


Table SVIII: Reaction barrier error for MS-PBE-in-PBE embedding with basis set truncation with respect to the full PBE calculations.

\begin{tabular}{lrrrrrrrr}
\hline \multicolumn{1}{c}{ Selection } \\
\hline Reaction & 1 & 2 & 3 & 4 & 5 & 6 & 7 & 8 \\
\hline$(\mathbf{1})$ & -0.065 & 1.243 & -0.017 & 1.233 & -0.009 & 1.260 & -0.000 & 0.949 \\
$(\mathbf{2})$ & 0.290 & -0.049 & -0.091 & -0.018 & -0.083 & 0.016 & -0.023 & -0.003 \\
$(\mathbf{3})$ & -1.691 & -0.206 & -0.088 & -0.058 & 0.171 & -0.016 & -0.005 & -0.003 \\
$(\mathbf{4})$ & -2.012 & -0.868 & -0.159 & 0.005 & - & - & - & - \\
\hline
\end{tabular}

\section{Manual Active-System Selection}

As an example to illustrate the subsystem partitioning for the manual selection (MS) Fig. S1 shows the case of the reactant of reaction (4). The subsystems are color-coded in the Lewis structure. Indices that reference subsystems start with the smallest active subsystem (1) and increase with the size of the active subsystem. The same color-code is used for all reactions.

In Fig. S2 the non-even-handed active-system selection for reaction (3) for the second manual selection is illustrated by showing a $\pi$-orbital of the pentacene molecule that is selected for reactant, transition state and product. As can be seen from the figure, the $\pi$-orbital selected for the transition state is not localized on the same atoms as in the reactant or product. A similar figure for reaction (4) and the largest (non-supersystem) selection is shown in Fig. S3 and for reaction (5) and the fourth selection in Fig. S4. 

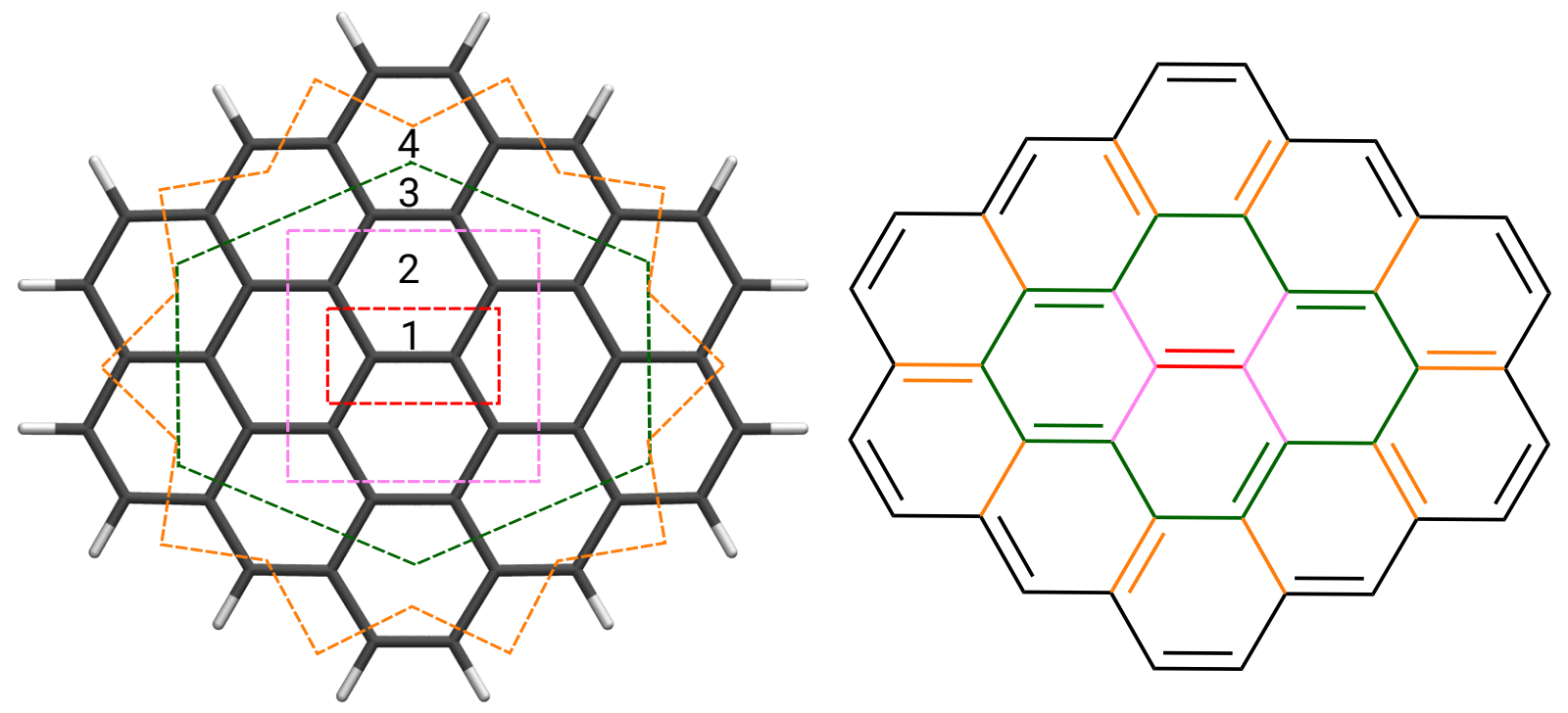

Figure S1: Illustration of the subsystem partitioning for the manual selection (MS) for reaction (4). The partitioning is color-coded in the lewis structure. Indices that reference a specific partitioning are counted from the smallest active subsystem (1) to larger indices $(2,3 \ldots)$. The same scheme applies to all reactions.

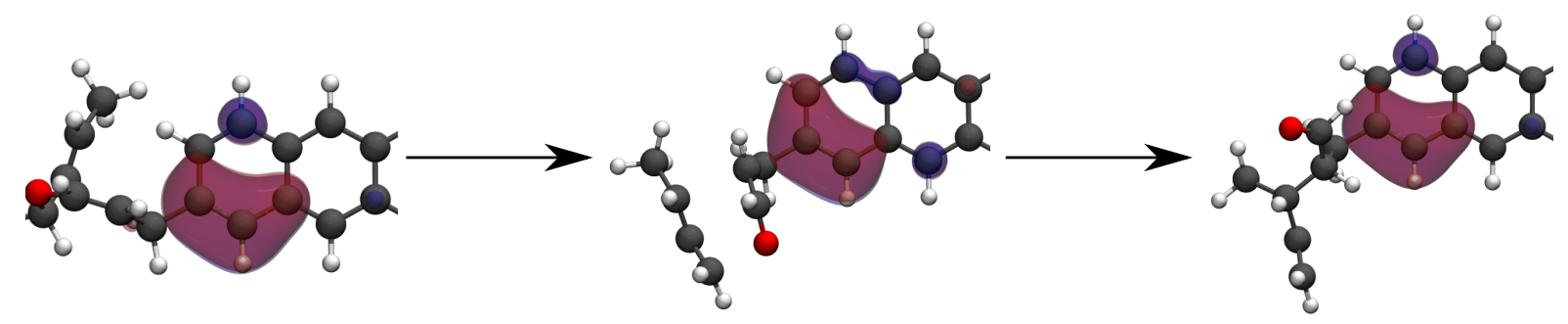

Figure S2: Isosurface plots of the one orbital selected as the active system for the manual selection approach for the second selection for reaction (3). The selection is based on the BLYP IBOs of the supersystem. Note that the selection is non-even-handed for the transition state with respect to reactant and product. The isosurface value for the orbitals was set to \pm 0.025 a.u. . 


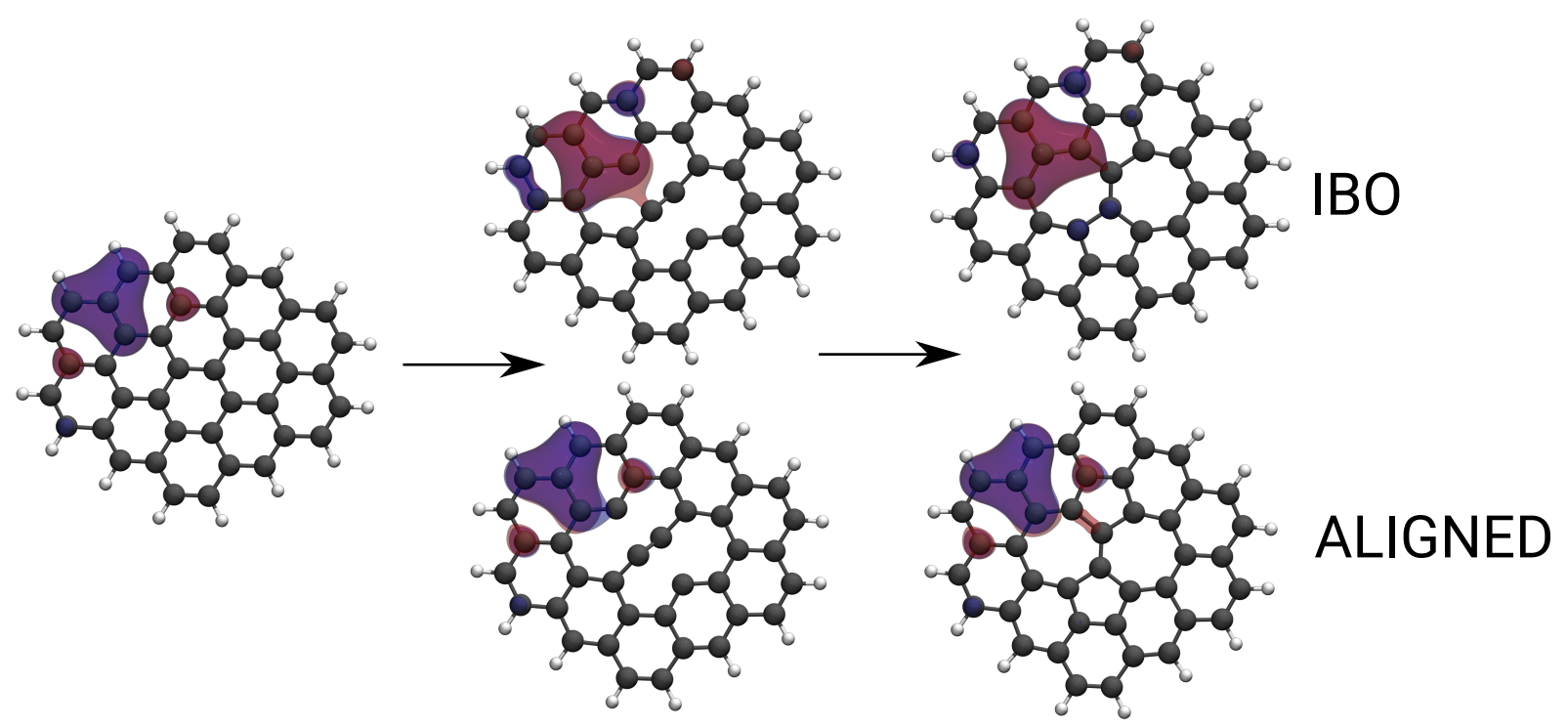

Figure S3: Isosurface plots of one orbital selected as the active system for the manual selection approach for the fourth selection for reaction (4). The selection is based on the BLYP IBOs of the supersystem. The aligned orbitals used in DOS are shown for comparison. The isosurface value for the orbitals was set to \pm 0.025 a.u. .

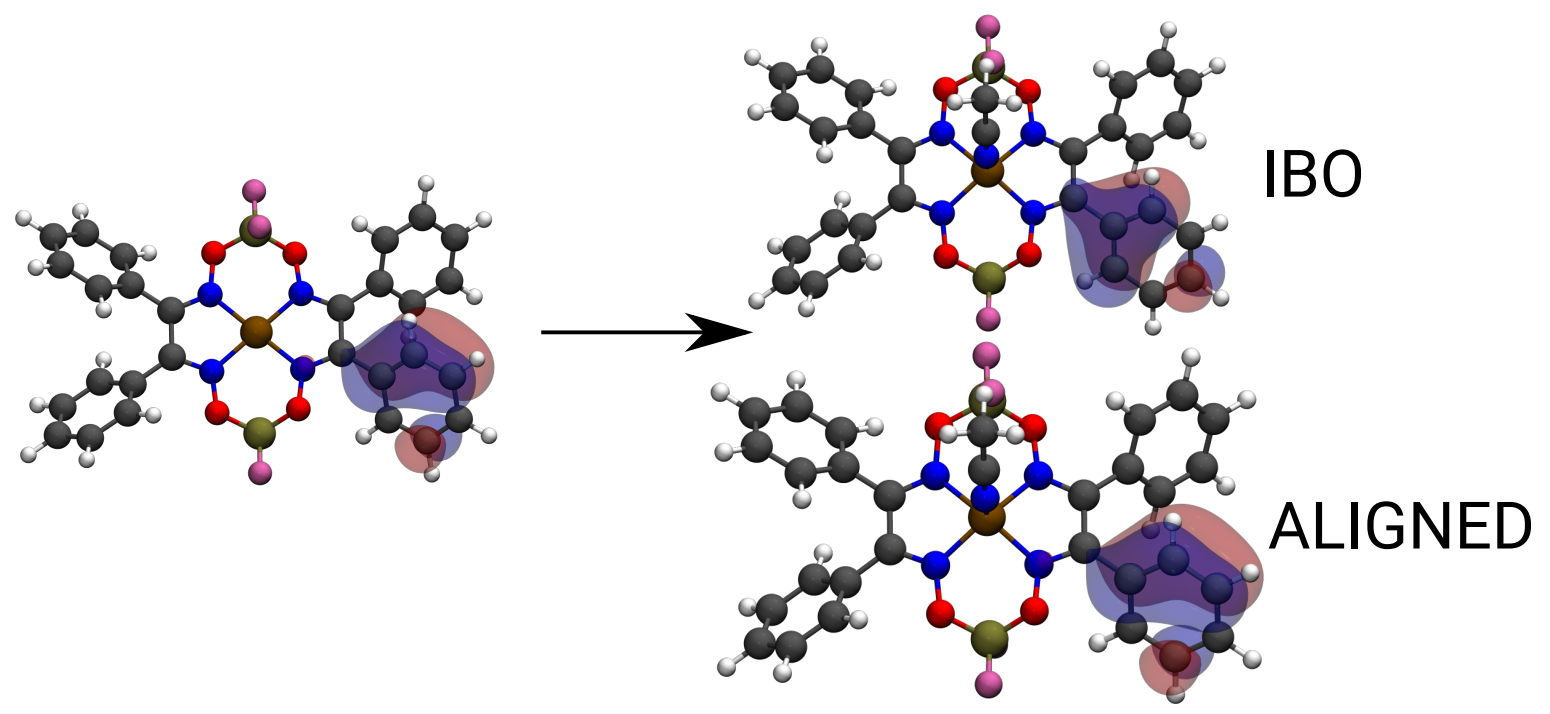

Figure S4: Isosurface plots of one orbital selected as the active system for the manual selection approach for the fourth selection for reaction (5). The selection is based on the BLYP IBOs of the supersystem. The aligned orbital used in DOS is shown for comparison. The isosurface value for the orbitals was set to \pm 0.025 a.u. . 


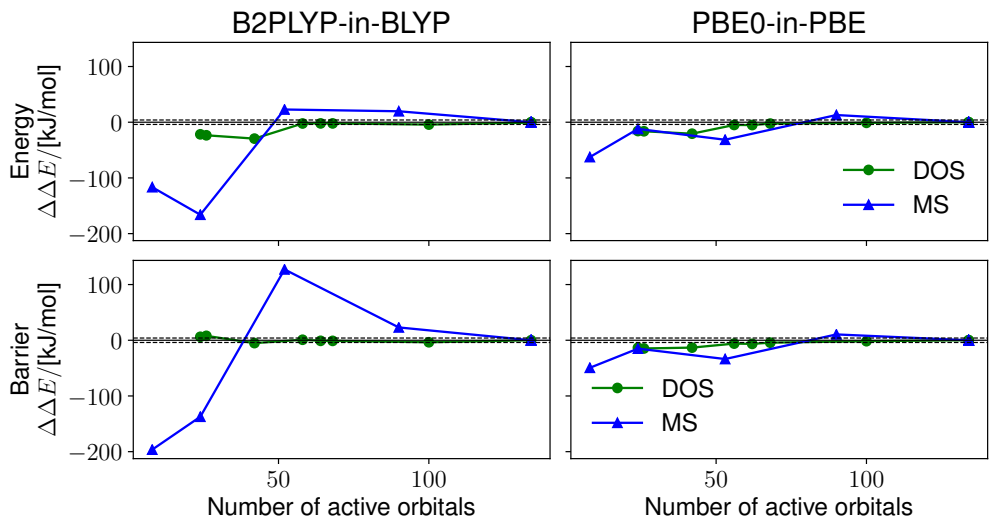

Figure S5: Errors in the reaction energy and barrier for the Stone-Waals defect formation (4) for $\mathrm{PbE}$ using $\mathrm{DOS}$ and MS. The reaction energy/barrier calculated is $308.91 \mathrm{~kJ} \mathrm{~mol}^{-1} / 907.50 \mathrm{~kJ} \mathrm{~mol}^{-1}$ with B2PLYP, $293.37 \mathrm{~kJ} \mathrm{~mol}^{-1} / 849.60 \mathrm{~kJ} \mathrm{~mol}^{-1}$ with BLYP, $310.00 \mathrm{~kJ} \mathrm{~mol}^{-1} / 944.33 \mathrm{~kJ} \mathrm{~mol}^{-1}$ with PBE0 and $292.91 \mathrm{~kJ} \mathrm{~mol}^{-1} / 869.42 \mathrm{~kJ} \mathrm{~mol}^{-1}$ with PBE. This full-scale graph corresponds to Fig. 9 of the main text.

\section{Full-scale Graphs}

The full-scale error graphs which correspond to the Figs. 9-11 of the main text are shown in Figs. S5, S6 and S7.

\section{Aligned and SPADE-based Manual Orbital Selection}

The reaction barriers calculated for B2PLYP-in-BLYP embedding using the active orbital selection based on singular value decomposition (SPADE) proposed by Claudino and Mayhall $^{1}$ and based on manual selection from the pre-aligned IBOs (aligned MS) used for the DOS are shown in Fig. S8 for reaction (3) and in Fig. S9 for reaction (4). The results obtained for non-aligned MS and DOS are shown for comparison. For aligned MS all active orbital space were always of the same size without needing to expand the size of one specific active orbital space as described in the main text. For the SPADEbased calculations the active orbital spaces were expanded based on the singular values associated to the orbitals if the active-orbital spaces were not of the same size. 


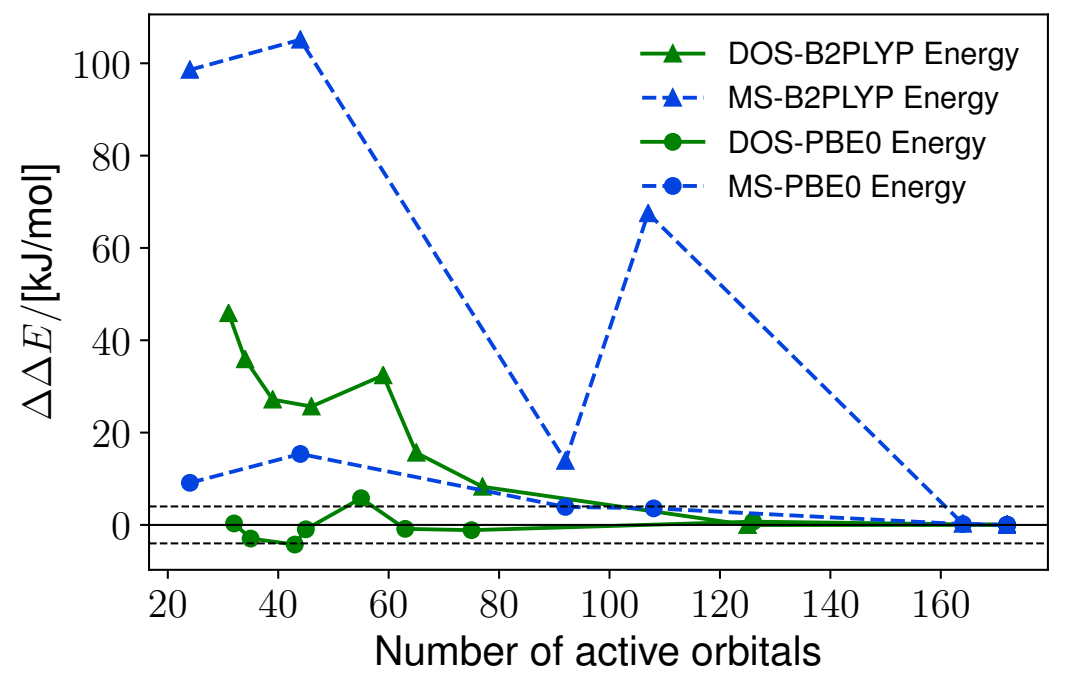

Figure S6: Errors in the reaction energy and barrier for the acetonitrile addition (5) for PbE using DOS and MS. The reaction energy calculated is $-105.11 \mathrm{~kJ} \mathrm{~mol}^{-1}$ with B2PLYP, $-66.26 \mathrm{~kJ} \mathrm{~mol}^{-1}$ with BLYP, $-56.43 \mathrm{~kJ} \mathrm{~mol}^{-1}$ with PBE0 and $-87.90 \mathrm{~kJ} \mathrm{~mol}^{-1}$ with PBE. This full-scale graph corresponds to Fig. 10 of the main text.

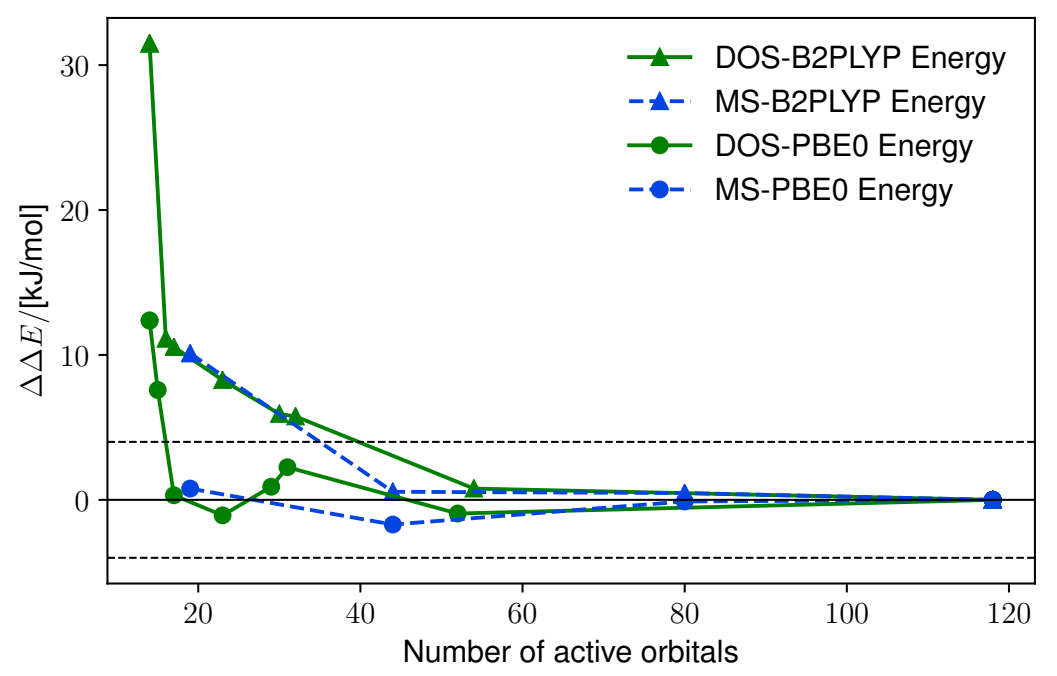

Figure S7: Errors in the reaction energy and barrier for the carbon monoxide addition (6) for PbE using DOS and MS. The reaction energy calculated is $-24.38 \mathrm{~kJ} \mathrm{~mol}^{-1}$ with B2PLYP, $-8.91 \mathrm{~kJ} \mathrm{~mol}^{-1}$ with BLYP, $-15.10 \mathrm{~kJ} \mathrm{~mol}^{-1}$ with PBE0 and $-16.81 \mathrm{~kJ} \mathrm{~mol}^{-1}$ with PBE. This full-scale graph corresponds to Fig. 11 of the main text. 

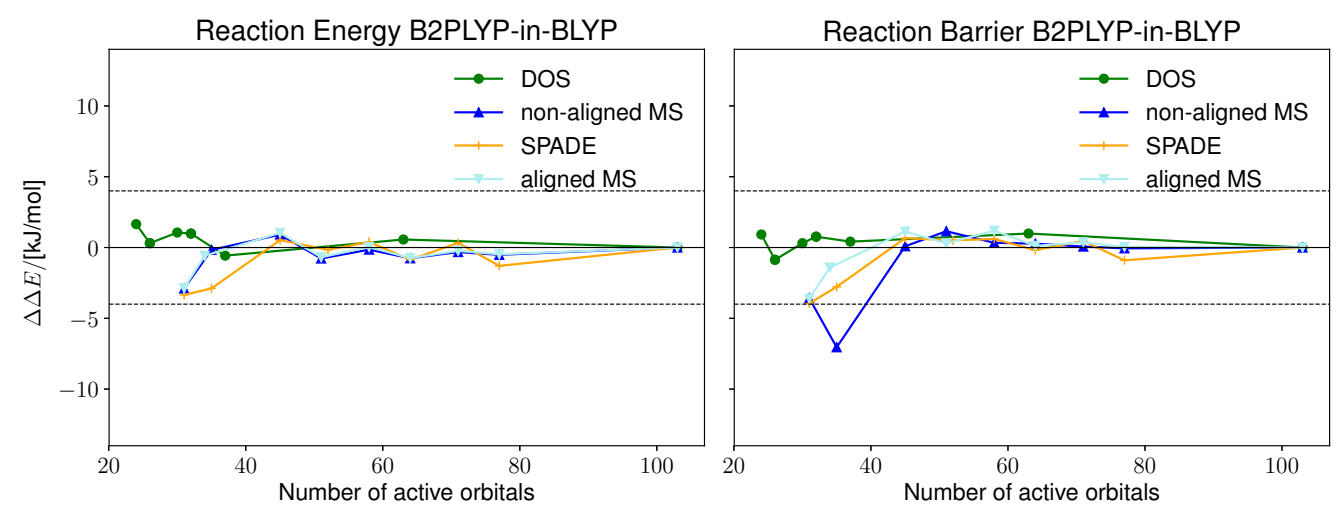

Figure S8: Errors in the reaction energy and barrier for the Claisen rearrangement (3) for PbE using DOS, SPADE and MS from non-aligned and aligned orbitals. The reaction energy/barrier calculated is $-72.70 \mathrm{~kJ} \mathrm{~mol}^{-1} / 106.19 \mathrm{~kJ} \mathrm{~mol}^{-1}$ with B2PLYP, $-53.71 \mathrm{~kJ} \mathrm{~mol}^{-1} / 87.87 \mathrm{~kJ} \mathrm{~mol}^{-1}$ with BLYP.
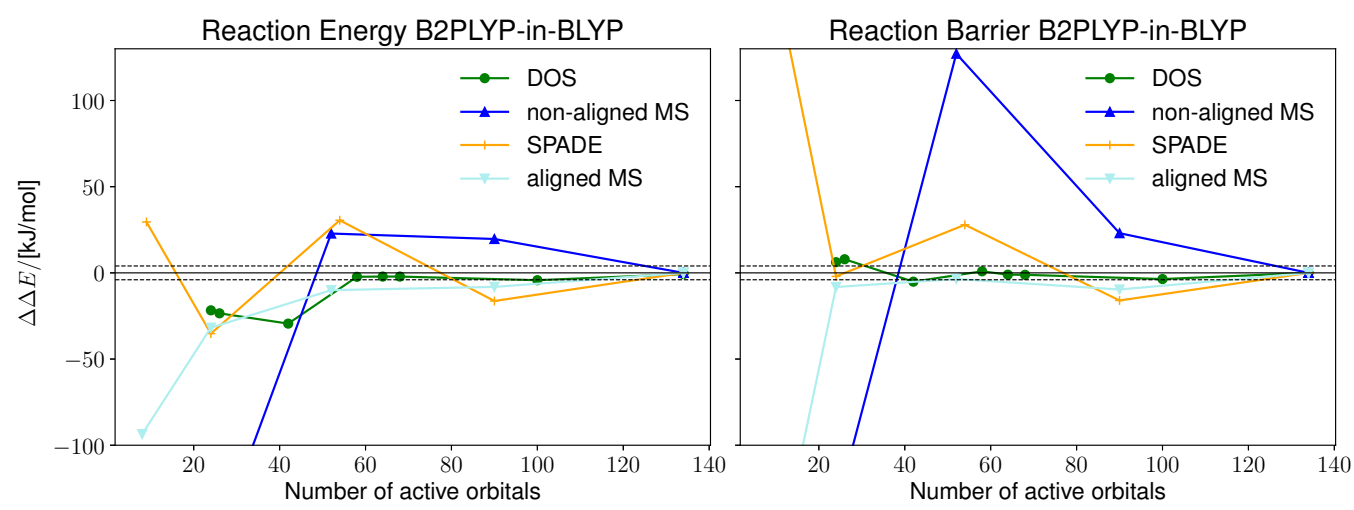

Figure S9: Errors in the reaction energy and barrier for the Stone-Waals defect formation (4) for PbE using DOS, SPADE and MS from non-aligned and aligned orbitals. The reaction energy/barrier calculated is $308.91 \mathrm{~kJ} \mathrm{~mol}^{-1} / 907.50 \mathrm{~kJ} \mathrm{~mol}^{-1}$ with B2PLYP, $293.37 \mathrm{~kJ} \mathrm{~mol}^{-1} / 849.60 \mathrm{~kJ} \mathrm{~mol}^{-1}$ with BLYP. 

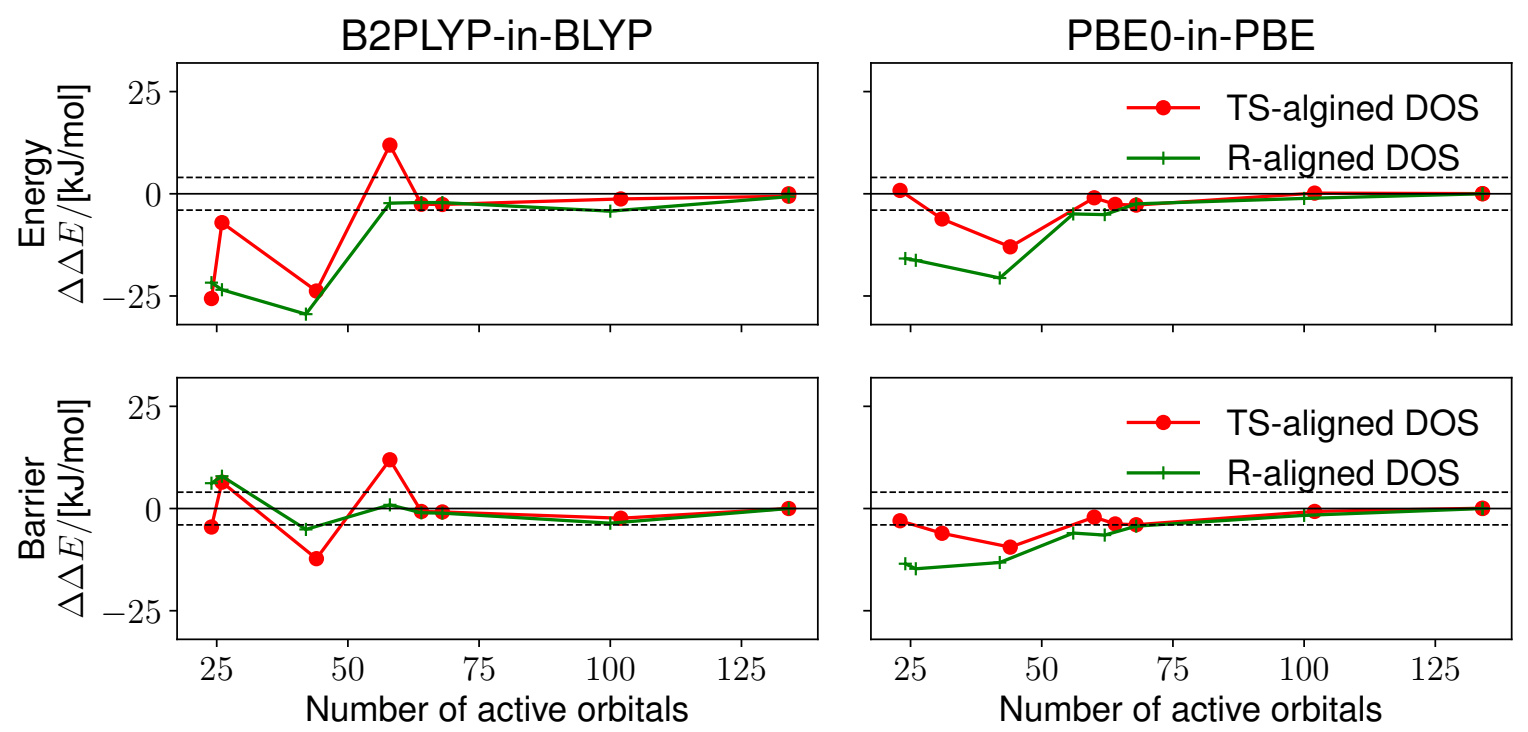

Figure S10: Errors in the reaction energy and barrier for the Stone-Waals defect formation (4) for $\mathrm{PbE}$ using $\mathrm{DOS}$ with orbitals that were aligned to the reactant $(\mathrm{R})$ or the transition state (TS) IBOs.

\section{Direct Orbital Selection from Transition State-aligned Orbitals}

The errors in reaction energy and barrier for the Stone-Waals defect formation (4) using the DOS with orbitals that were initially aligned to the IBOs of the transition state or the reactant are shown in Fig. S10.

\section{Domain-based Local Pair Natural Orbital Coupled Cluster Implementation}

Our implementation of the domain-based local pair natural orbital coupled cluster with single, double and perturbative triple excitations [DLPNO-CCSD $\left.\left(\mathrm{T}_{0}\right)\right]$ is based on the equations given in the appendix of Ref. 2. The pair-prescreening, integral transformation 
and pair-natural orbital (PNO) construction is based on Refs. 3,4. The triples correction is calculated in a semi-canonical fashion as documented in Ref. 5. The source-code will be provided in a future release of the quantum-chemistry program package SERENITY ${ }^{6}$ which is publicly available online (https://github.com/qcserenity/serenity).

Table SIX: Correlation energies calculated with DLPNO-CCSD $\left(\mathrm{T}_{0}\right)$ with the quantum chemistry program packages SERENITY and ORCA for reactions (2), (3) and (6). The calculations marked with * are performed with "TightPNO" settings in ORCA. All other calculations are performed with "NormalPNO" settings in ORCA and corresponding settings in SERENITY.

\begin{tabular}{|c|c|c|}
\hline & \multicolumn{2}{|c|}{ Correlation Energy in $\mathrm{E}_{\mathrm{h}}$} \\
\hline & SERENITY & ORCA \\
\hline \multicolumn{3}{|c|}{ Diels-Alder reaction (2) } \\
\hline Product & -3.29690 & -3.29662 \\
\hline Reactant & -3.28399 & -3.28349 \\
\hline Transition state & -3.32089 & -3.32052 \\
\hline \multicolumn{3}{|c|}{ Claisen rearrangement (3) } \\
\hline Product & -4.38957 & -4.38868 \\
\hline Reactant & -4.38315 & -4.38215 \\
\hline Transition state & -4.41319 & -4.41203 \\
\hline \multicolumn{3}{|l|}{ CO-Addition (6) } \\
\hline Product & -5.02318 & $-5.01500 /-5.02333^{*}$ \\
\hline Reactant & -5.00942 & $-5.00151 /-5.00992^{*}$ \\
\hline
\end{tabular}

A comparison of correlation energies calculated with ORCA and SERENITY is given in Tab. SIX for reactions (1), (3) and (6). The Def2-SVP basis set and the intrinsic bond orbital (IBO) localization scheme were used. The differences in the correlation energies calculated with ORCA and SERENITY are well below $0.1 \%$ of the correlation energies. 
To put this into perspective, this is the accuracy typically aimed at by DLPNO-CCSD compared to the canonical coupled-cluster calculation ${ }^{4}$ (the semi-canonical, non-orbital invariant-triples correction recovers typically $91-94 \%$ of the canonical-triples correction ${ }^{5}$ ). Note that the ORCA results calculated with "NormalPNO" settings for reaction (6) deviate somewhat more significantly from the results calculated with equivalent settings with SERENITY. However, if "TightPNO" settings are used in ORCA, the correlation-energy calculated by SERENITY is reproduced up to the desired accuracy. 


\section{References}

[1] Claudino, D.; Mayhall, N. J. Automatic partition of orbital spaces based on singular value decomposition in the context of embedding theories. J. Chem. Theory Comput. 2019, 15, 1053-1064.

[2] Werner, H.-J.; Schütz, M. An efficient local coupled cluster method for accurate thermochemistry of large systems. J. Chem. Phys. 2011, 135, 144116.

[3] Pinski, P.; Riplinger, C.; Valeev, E. F.; Neese, F. Sparse maps-A systematic infrastructure for reduced-scaling electronic structure methods. I. An efficient and simple linear scaling local MP2 method that uses an intermediate basis of pair natural orbitals. J. Chem. Phys. 2015, 143, 034108.

[4] Riplinger, C.; Pinski, P.; Becker, U.; Valeev, E. F.; Neese, F. Sparse maps-A systematic infrastructure for reduced-scaling electronic structure methods. II. Linear scaling domain based pair natural orbital coupled cluster theory. J. Chem. Phys. 2016, 144, 024109 .

[5] Riplinger, C.; Sandhoefer, B.; Hansen, A.; Neese, F. Natural triple excitations in local coupled cluster calculations with pair natural orbitals. J. Chem. Phys. 2013, 139, 134101.

[6] Unsleber, J. P.; Dresselhaus, T.; Klahr, K.; Schnieders, D.; Böckers, M.; Barton, D.; Neugebauer, J. Serenity : A subsystem quantum chemistry program. J. Comput. Chem. 2018, 39, 788-798. 